\title{
Questions de communication
}

Serge Tisseron, Sylvain Missonnier, Michael Stora, L'enfant au risque du virtuel

Paris, Dunod, 2006

Nicolas Casel

\section{OpenEdition}

\section{Journals}

Édition électronique

URL : http://journals.openedition.org/questionsdecommunication/1234

DOI : 10.4000/questionsdecommunication. 1234

ISSN : 2259-8901

\section{Éditeur}

Presses universitaires de Lorraine

Édition imprimée

Date de publication : 1 juillet 2009

Pagination : 504-506

ISBN : 978-2-86480-989-0

ISSN : 1633-5961

\section{Référence électronique}

Nicolas Casel, « Serge Tisseron, Sylvain Missonnier, Michael Stora, L'enfant au risque du virtuel », Questions de communication [En ligne], 15 | 2009, mis en ligne le 18 janvier 2012, consulté le 21 septembre 2020. URL : http://journals.openedition.org/questionsdecommunication/1234 ; DOI https://doi.org/10.4000/questionsdecommunication.1234

Ce document a été généré automatiquement le 21 septembre 2020

Tous droits réservés 


\title{
Serge Tisseron, Sylvain Missonnier, Michael Stora, L'enfant au risque du virtuel
}

Paris, Dunod, 2006

\author{
Nicolas Casel
}

\section{RÉFÉRENCE}

Serge Tisseron, Sylvain Missonnier, Michael Stora, L'enfant au risque du virtuel. Paris, Dunod, coll. Inconscient et culture, 2006, 200 p.

1 Apparu au grand public de manière tangible au début des années 90, le virtuel est de nos jours un objet dont la définition n'est pas figée, et dont l'exploration n'a pas encore livré tous ses résultats. Et pour cause, l'une de ses caractéristiques majeures est d'être en constante transformation. Un collectif d'auteurs - Serge Tisseron, psychiatre et psychanalyste, en tête, avec Sylvain Missonnier, maître de conférences en psychologie clinique, et Michael Stora, psychologue-psychanalyste - se propose de fournir un décryptage des relations entre l'enfant et la machine, dans ses usages quotidiens des nouvelles technologies.

Dans cet ouvrage, différentes théories, issues en particulier du champ de la psychologie, éclairent l'objet qu'est le virtuel en insistant sur les pouvoirs de l'image et le rôle qui lui est conféré. En effet, l'image est avant tout synonyme de signification, d'enveloppement et de transformation. En conséquence, elle en devient un espace de symbolisation, renforcé par le fait qu'elle est aussi le lien entre le monde interne de l'enfant, et le monde externe. En parallèle, le facteur "risque», auquel le terme "tentation » pourrait être préféré, est mis en perspective par le désir incessant qu'a l'être humain, dès sa naissance, de vouloir se confondre avec l'objet de son attachement, et en même temps de vouloir s'en séparer. Inévitablement, il naît un conflit entre s'unir à l'objet, qui fait alors craindre de s'y perdre, et se détacher de 
l'objet qui provoque la peur de la solitude ;ce dilemme fait directement référence au positionnement de chacun par rapport aux images. De plus, le risque est évoqué aussi du point de vue des nouvelles formes de socialisation, et donc de désocialisation; les auteurs s'attachant à décrire les difficultés posées par ces nouveaux usages en termes de construction de repères et d'identité.

En somme, le collectif d'auteurs apporte des éléments de réponse aux questions que beaucoup se posent, à propos des changements de perception que chacun a de luimême, changements produits par les mondes virtuels que composent Internet et les jeux vidéo. Premiers concernés par cette problématique du rapport au virtuel, les adolescents, puisque ce sont eux les premiers "consommateurs" des nouvelles technologies. Véritables « enfants des nouveaux médias» (p. 2), ils consacrent de plus en en plus de temps à l'internet, au point que les parents s'inquiètent de potentielles addictions, déconnexions du réel, ou même pathologies. Serge Tisseron met d'abord en avant la nécessité d'un accompagnement des parents et des éducateurs pour une appropriation puis une utilisation dynamique et raisonnée des univers virtuels, puis il souligne le possible lien entre les failles, apparues lors du développement psychologique de l'enfant et les usages pathologiques de ces technologies. Pour sa part, Sylvain Missonnier démontre que la relation à l'objet virtuel peut se réaliser au quotidien, et ce de manière inconsciente; il s'appuie sur l'exemple de l'échographie fœtale pour illustrer son propos. De son côté, Michael Stora prouve que c'est bien le cadre et la pratique autour de l'objet virtuel, et non pas l'objet en tant que tel, qui peuvent engendrer des usages à problèmes, puis il démontre, situations réelles à l'appui, les effets thérapeutiques du jeu vidéo.

Si un minimum de culture numérique s'avère nécessaire pour saisir le propos global du livre, la vulgarisation des théories scientifiques et la clarté de sa structure le destinent aussi bien à un public néophyte dans le domaine de la psychologie, qu'à des enseignants ou des chercheurs en quête d'une étude objective sur le rapport de l'enfant au virtuel. Pour les parents soucieux d'en comprendre les enjeux, ils seront en mesure de se faire un avis critique, à partir des nombreux exemples concrets, en partie tirés de cas réels. Un bémol cependant, la partie rédigée par Sylvain Missonnier n'est pas aussi accessible, car elle demande des connaissances en psychologie qui relèvent clairement du niveau universitaire. Dans le même ordre d'idée, mais à un degré moindre, Michael Stora décrit des aspects précis de certains jeux vidéo qui ne trouveront un écho que chez les lecteurs imprégnés d'une culture vidéoludique certaine.

Le livre est relativement court (200 pages) et se décompose en quatre grandes parties. Dans un premier temps, c'est au travers des jeux vidéo que Serge Tisseron explique la fascination pour le virtuel. D'après lui, le joueur se pose quatre questions qui renvoient à quatre moments essentiels de la vie: la découverte de nouvelles sensations, la manipulation des objets, la construction et la reconnaissance de sa propre valeur, et enfin l'angoisse de la rencontre. Comme le bébé est plongé dans un bain d'excitations, le joueur cherche à s'immerger dans le virtuel, mais cette fois-ci en vue de maitriser et de donner du sens à ces excitations. Lors du deuxième moment, de par ses possibilités de "pâte à modeler numérique " (p.12), le jeu vidéo apparaîtrait comme un objet transitionnel chez Donald Winnicott (De la pédiatrie à la psychanalyse, trad. de l'anglais revue, annotée et augmentée par Jeannine Kalmanovitch, Paris, Payot, 1958 [1969]) ou un objet malléable chez René Roussillon (Paradoxes et situations limites de la psychanalyse, Paris, Presses universitaires de France, 1991). Plus tard, c'est un gain narcissique qui 
s'opère chez le joueur, grâce aux interactions avec d'autres joueurs ou internautes, lui apportant des gratifications et de multiples retours qui prouvent que les aspects de soi, a priori peu avantageux, ne sont pas aussi dramatiques qu'il le pense. Enfin, la rencontre avec autrui permet d'avoir le contrôle de la dynamique fusion- séparation, en engageant ou en mettant fin à une relation d'un simple clic. Le psychanalyste va plus loin en dressant, à partir de ces quatre étapes, une typologie du joueur, et en attribuant un genre de jeu favori à chacun : par exemple, ceux qui préfèrent manipuler les objets vont plus s'orienter vers des jeux de construction, de stratégie, ou de simulation de vie.

La deuxième partie expose la «psycho(patho)logie psychanalytique du virtuel quotidien» (p. 39) de Sylvain Missonnier. Son postulat est que les objets banals sont producteurs de représentations individuelles et collectives, alors qu'en règle générale ils ne sont pas du tout pris en considération par la recherche, en particulier les objets techniques. Il prend comme exemple précis l'échographie obstétricale qui met en rapport un objet technique avec un processus psychique; ce cas de figure s'avère pertinent, d'abord pour examiner dans quelle mesure la «virtualisation échographique» (p.53) peut s'avérer bénéfique ou à l'inverse délétère pour le processus de parentalité, puis pour proposer une psycho(patho)logie du virtuel quotidien.

7 Dans la troisième partie de l'ouvrage, Serge Tisseron commence par évoquer le mythe de la caverne de Platon, à propos du monde des Idées auquel les humains n'ont jamais accès et qui se contentent de croire aux apparences. En s'appuyant sur les films Matrix, des frères Wachowski, et Avalon, de Mamoru Oshii, il explique que ce mythe est toujours d'actualité dans la mesure où la "réalité virtuelle correspond donc à ce qu'on aurait appelé en d'autres temps un royaume des apparences. Les humains qui y évoluent l'ignorent» (p. 88). L'idée est que nos relations avec les univers virtuels sont semblables à celles que nous avons avec les images. En effet, il souligne la correspondance entre ces deux films et nos deux positions face aux images : nous aimerions soit qu'elles nous absorbent, soit y pénétrer pour les contrôler. Par la suite, l'auteur propose le virtuel en tant que relation. À travers ses différentes facettes, qu'il se donne comme «en puissance ", comme " non actualisé » ou comme « refus du corporel », le virtuel renvoie aux aspirations des adolescents. Effectivement, le développement psychologique de l'adolescence répond de manière presque parfaite aux avantages du virtuel que sont le sentiment d'immersion, l'interactivité et la rencontre avec l'autre.

8 Enfin, Michael Stora décrit les effets thérapeutiques du jeu vidéo. Animateur d'un atelier jeu vidéo au Centre médico-pédagogique CMP de Pantin, il aide les enfants en difficultés psychologiques à exprimer leurs émotions, accepter et dépasser leurs blocages psychiques, par la pratique des jeux. Le rôle de ces derniers est de permettre de faire agir et surtout d'interagir avec une représentation de soi à l'écran; ce qui correspond aux attentes d'un public qui surinvestit les images dès les premières années de la vie. Concrètement, dans un cadre de narration, et avec le suivi attentif d'un psychologue, jouer contribue à une lutte antidépressive grâce à la mise en jeu de pulsions agressives, améliore l'estime de soi en incarnant des figures héroïques et en atteignant des objectifs imposés par l'ordinateur. Ainsi Michael Stora légitime-t-il cette forme de thérapie car « l'espace virtuel leur donne accès à leur inconscient et le rôle du thérapeute est donc d'aider par sa présence à ce qu'ils y accèdent, même si cela passe par un scénario agressif» (p. 157). Avec pour exemples les jeux Ico, Halo et Les Sims, l'auteur explique en quoi chacun d'eux peuvent révéler, grâce à leur gameplay et leur 
scénario propres, certains processus psychologiques du joueur. Par exemple, on retiendra la possibilité qu'ont les enfants de revivre et d'achever la phase de séparation dans leur relation à la mère, en jouant à la simulation de vie Les Sims.

9 Le message global de l'ouvrage est bien de nuancer les prétendus dangers du virtuel. Sur ce plan, Serge Tisseron estime « qu'au-delà du caractère parfois excessif de chacune de ces pratiques, une nouvelle culture est en train de s'inventer » (p. 167). En effet, les nouvelles technologies amènent un changement profond dans le développement psychologique et social des enfants, puisque leur utilisation porte à conséquence sur le rapport à l'image, la gestion des conflits psychiques, le passage à l'âge adulte et la définition de l'identité, voire de l'intimité.

\section{AUTEURS}

\section{NICOLAS CASEL}

CREM, université Paul Verlaine-Metz

nicolas.casel@aromate.org 